\title{
Brain tissue echogenicity-implications for substantia nigra studies in parkinsonian patients
}

\author{
Krzysztof Sadowski • Karol Szlachta • \\ Małgorzata Serafin-Król · Jolanta Gałązka-Friedman • \\ Andrzej Friedman
}

Received: 6 May 2011/ Accepted: 20 August 2011/Published online: 1 September 2011

(c) The Author(s) 2011. This article is published with open access at Springerlink.com

\begin{abstract}
The aim of the present study was to assess the origin of the substantia nigra hyperechogenicity in Parkinson disease patients. The cause of hyperechogenicity was tested on an animal model. Fresh porcine brains were injected consecutively with ferritin, apoferritin and water. Then, glioma samples were inserted into animal model. The echogenicity of the region of interest was assessed before and after experimental procedures. We observed the same echogenicity of porcine brain before and after injections of iron-loaded ferritin, apoferritin and water. Increased echogenicity of glioma samples compared to surrounding porcine brain tissue could be clearly seen. We postulate that the relative gliosis might be, at least partially, responsible for the increased echogenicity of the substantia nigra in Parkinson disease patients. Keeping in mind all limitations and inaccuracies of animal model used, it seems that hyperechogenicity of substantia nigra is caused rather by structural changes within the brain tissue than by increased iron concentration.
\end{abstract}

K. Sadowski and K. Szlachta contributed equally to the study.

K. Sadowski $(\bowtie) \cdot$ A. Friedman

Department of Neurology, Health Science Faculty,

Medical University of Warsaw, Kondratowicza 8,

03-242 Warsaw, Poland

e-mail: sadowski.ks@gmail.com

K. Szlachta · J. Gałązka-Friedman

Faculty of Physics, Warsaw University of Technology,

Warsaw, Poland

M. Serafin-Król

Department of Diagnostic Imaging, IInd Medical Faculty,

Medical University of Warsaw, Warsaw, Poland
Keywords Transcranial sonography - Iron · Ferritin · Parkinson's disease · Substantia nigra hyperechogenicity

\section{Introduction}

Differential diagnosis of Parkinson's disease (PD) and atypical parkinsonian syndromes is based mainly on clinical examination (Hughes et al. 2002). Transcranial sonography (TCS) could serve as easily accessible, quick and safe diagnostic tool for movement disorders neurologist. Recent studies indicate that midbrain abnormalities determined by TCS might be useful findings in uncertain cases of parkinsonism (Becker et al. 1995; Berg et al. 1999a; Walter et al. 2003). Becker et al. postulated that the relative increase of glial cells concentration and microstructural changes in the $\mathrm{SN}$ may result in increased tissue echogenicity. Further studies concentrated on the role of iron in the hyperechogenicity of SN (Berg et al. 1999b, 2002; Zecca et al. 2005). Berg et al. injected rat brains with ionic iron, ferritin, 6-OHDA together with desferrioxamine, zinc and buffer into the SN. A dose-dependent increase of SN echogenicity was observed in the case of ionic iron injection only. In another study, Zecca et al. (2005) scanned post-mortem human brains at different ages. The correlation was observed between SN echogenicity and the concentration of iron measured by atomic absorption spectroscopy as well as between SN hyperechogenicity and $\mathrm{H}-$ and L-ferritins. The exact role of iron in parkinsonian neurodegenerative process needs to be elucidated. Accurate quantification of iron levels in control and PD brains (Wypijewska et al. 2010) shows that total iron level remains unchanged and only labile iron level increases. The concentration of labile iron is almost 2,000 times smaller than the concentration of total iron. In most 
recent studies (Berg et al. 2010), the role of activated microglia in substantia nigra hyperechogenicity was emphasized again. The aim of this study was to assess the influence of iron-loaded ferritin and glial tissue on echogenicity of the brain structures.

\section{Methods}

The study does not involve intervention for research purposes or identifiable patient data.

Ultrasound imagining technique

Experiment was performed on Esaote MyLab 70XVision ultrasound scanner equipped with 13-4 MHz Linear Array probe working in $\mathrm{B}$ mode.

\section{Animal model}

The cause of hyperechogenicity was tested on an animal model (Fig. 1). Fresh, i.e. max $8 \mathrm{~h}$ after animal death, porcine brains were used. No method of tissue fixation was applied for the experiment. To obtain the homogeneous structure, 3-4 brains were tightly put into the plastic holder for each measurement. Brain tissue was consecutively covered with skin and $5 \mathrm{~mm}$ layer of fat. Skin and fat absorb ultrasounds similarly to skull. Special attention was paid to remove all air bubbles, so free spaces were filled with USG gel. In this study we did not aim to identify particular brain structures. We used porcine brains as a medium similar to human brain tissue for experimental procedures. The experimental model we used was maximally simplified and, simultaneously, designed to mimic conditions similar to those in real patients. It is not possible to localize $\mathrm{SN}$ or any other particular anatomical structure in such conditions. In spite of that, as described further in the study, we were able to assess repeatable conditions and well established localization for experimental procedures. Ferritin (Sigma Aldrich horse spleen ferritin) of about $1 \mathrm{~cm}^{3}$ volume, containing 4,000 $\pm 200 \mu \mathrm{g} / \mathrm{g}$ of iron (the concentration of iron was assessed by us with atomic absorption), apoferritin (ferritin void of iron, Sigma Aldrich horse spleen ferritin) also of about $1 \mathrm{~cm}^{3}$ volume, containing $4.0 \pm 0.4 \mu \mathrm{g} / \mathrm{g}$ of iron and $1 \mathrm{~cm}^{3}$ volume water were injected, respectively, into our models and ultrasound examination was performed for each injection separately. Injection depth was $1.5-2 \mathrm{~cm}$. In the next experiment, samples of glial tissue obtained from human glioma (approx. $0.2 \mathrm{~cm}^{3}$ samples of astrocytoma GII and glioblastoma multiforme GIV, stored at $-70^{\circ}$, without tissue fixation procedures before the experiment) after neurosurgical operation were inserted into porcine brain and USG was performed.

Region of interest was always marked either with the top of injection needle in case of injections or with metal grid for experiment with glial tissue. The localization of the injection needle seen as the regular linear structure producing intensive echo could be unequivocally determined. Therefore, we were able to assess repeatable conditions for experimental infusions. In the first stage of the experiment, the change of tissue echogenicity after ferritin, apoferritin and water infusion was assessed. The echogenicity shift at the site of the injection needle tip was visually evaluated at the time and directly after the infusion. Then the pictures of the ROI were also assessed by the same experienced sonographist, to obtain better reliability. The TCS with glioma tissue insertions was performed as next part of our experiment with the use of the same porcine brain model.
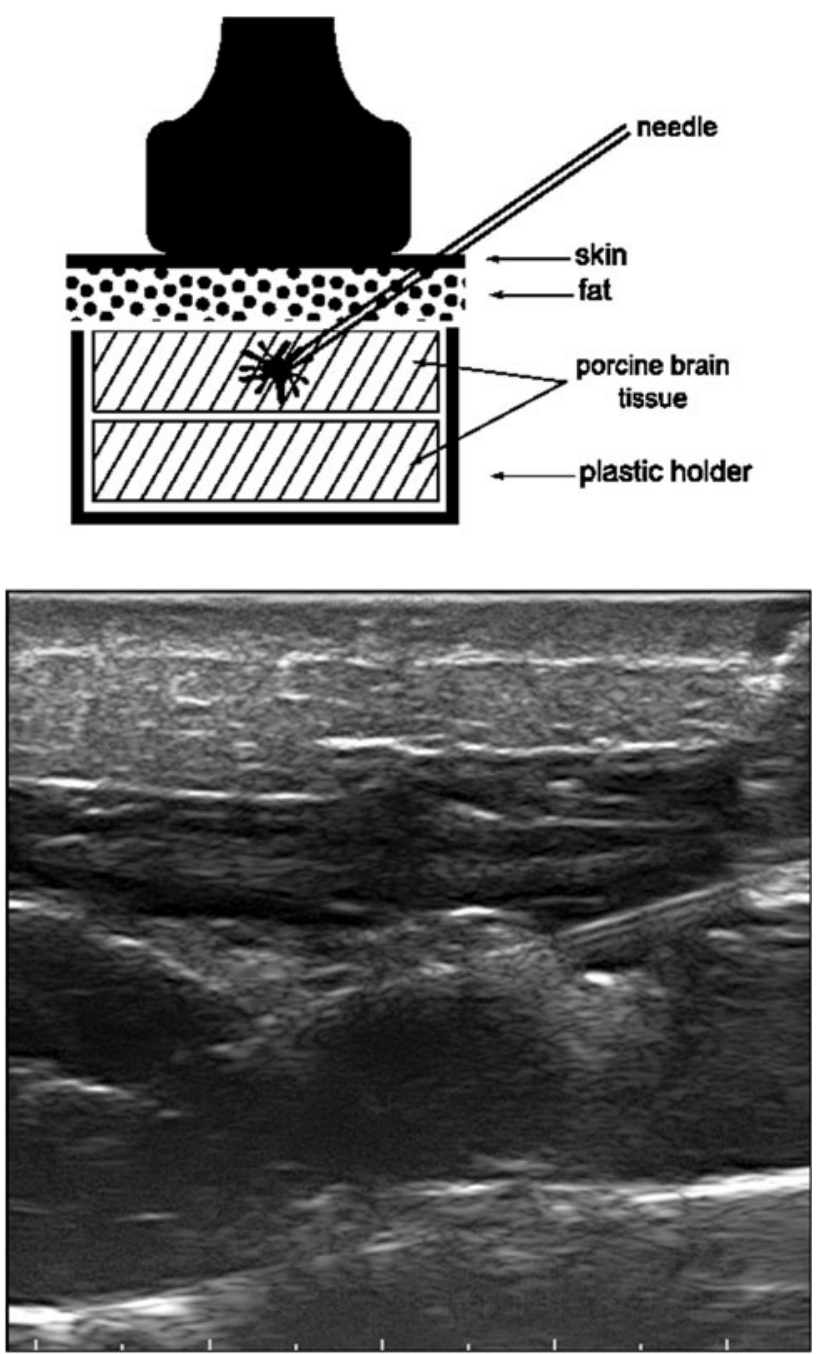

Fig. 1 Animal model scheme and its photographic registration. Injection needle can be seen on the right. Skin and fat on top-first bright layer 
For better localization, tumour samples were inserted in the centre of the metal grid and then tightly covered with brain tissue as well as fat and skin layer. The ultrasound examination was performed before and after glioma insertion. Photographic registration was also made and the images were carefully assessed by experienced sonographist.

\section{Quantification of echogenicity}

The change of tissue echogenicity after injections and glial tissue insertions was assessed qualitatively by experienced physician during model examinations.

\section{Results}

As seen in Fig. 1, all layers of animal model (i.e. fat plus skin layer, porcine brain tissue, plastic holder wall) were clearly depicted. Figure $2 \mathrm{a}, \mathrm{b}$, c shows the same echogenicity of porcine brain before and after injections of ironloaded ferritin (a), apoferritin (b) and water (c). We were unable to notice any change in tissue echogenicity except subtle transient distortions caused by liquid flow, which were the same as typical for any liquid infusion. No stable change was observed.

On the other hand, in the second part of our experiment a marked hyperechogenicity in the area of glioma sample insertion was noticed (Fig. 2d). There was no significant difference between GII and GIV samples.

\section{Discussion}

By definition, tissue echogenicity describes its ability to create echo during ultrasound examination. In our study, we were focused only on brightness change caused by different factors. In clinical trials assessing substantia nigra and other basal ganglia echogenicity in parkinsonian patients, the area of hyperechogenic region is taken into consideration. There is no accepted and standardized method of tissue echogenicity assessment. Our experiment has shown that ferritin overloaded with iron is not able to cause brain tissue hyperechogenicity. It is important to note that the concentration of iron in the ferritin injected in our experiment was even higher than that in human substantia nigra. Observed effect was similar to those of apoferritin or water injection. There was no visible change in tissue echogenicity in the region of interest. Should the hyperechogenicity of the SN in PD patients depend on iron overload, injections of iron-loaded ferritin would have to produce such an effect. Having in mind the dense structure of the experimental model, physical rules of liquid diffusion and the fact, that photographic registration was performed immediately after infusions, it appears to be improbable, that the infused liquids could leave the region of interest and penetrate the surrounding tissue at the time of experiment.

Our findings are, at least partially, in contradiction to findings of Berg et al. (1999b). In our study, we did not use living brains at any stage of the experiment. The measurements were obtained immediately after experimental injections, so, most probably, we were able to observe the effect of injected substances "in situ" and in real time before any biochemical interactions between injected substances and surrounding tissue occurred. In the study by Berg et al., experimental solutions were injected into the $\mathrm{SN}$ of the living rat brain. Animals were killed 1 week after stereotactic injection. Ultrasound examination and biochemical analysis were performed post-mortem. Echogenicity of the injected SN increased with the concentration of iron injected. At $1.5 \mu \mathrm{g}$ not only SN but also adjacent brain structures were hyperechogenic. At $7.5 \mu \mathrm{g}$ the echogenicity of the whole brain hemisphere increased. In our opinion, this massive increase of ionic iron in SN and its consequence is far from conditions met in SN of parkinsonian patients. Obviously, such a massive metal solution injection would change tissue echogenicity. One has to underline that after zinc injection, hyperechogenicity of the whole hemisphere was also seen. Undoubtfully metal injection changed tissue echogenicity. We do not know, however, about the exact cause of this hyperechogenicity (no histopathological data available). The question remains what was going on within the injected hemisphere during the week between injection and further experimental procedures. Iron injection might have caused inflammatory glial response which might account for tissue hyperechogenicity. This subject needs further research. Having this in mind our own findings and findings of Berg et al. might be only partially (if at all) conflicting.

One has to bear in mind that SN hyperechogenicity is not pathognomonic for PD. About $10-15 \%$ of MSA and PSP patients are characterized by the same ultrasound feature. There is also a relatively high percentage (upto $80 \%$ ) of patients with Lewy bodies dementia and corticobasal syndrome having hyperechogenicity of the SN (Walter et al. 2006). The role of iron metabolism disequilibrium in these particular neurodegenerative diseases remains unclear, whereas neuronal loss and reactive gliosis are obvious. One should mention that in physiological conditions there are, except for the $\mathrm{SN}$, other iron-rich structures in the brain such as globus pallidus (GP) and red nucleus $(\mathrm{RN})$. These are non-detectable (GP) or variably detectable (RN) by TCS. On the contrary, increased echogenicity of glioma samples compared to surrounding brain tissue could be clearly seen. There was no significant difference between GII and GIV samples. It is obvious that 
Fig. 2 a Left plain animal model. Right same model after ferritin injection. Injection needle can be seen. b Left plain animal model. Right same model after apoferritin injection on the right. Injection needle can be seen. c Left plain animal model. Right same model after water injection. d Left plain animal model. Right same model with glioma inserted. Needle (see interference pattern) marked tissue insertion. Hyperechogenicity compared to plain model can be clearly seen
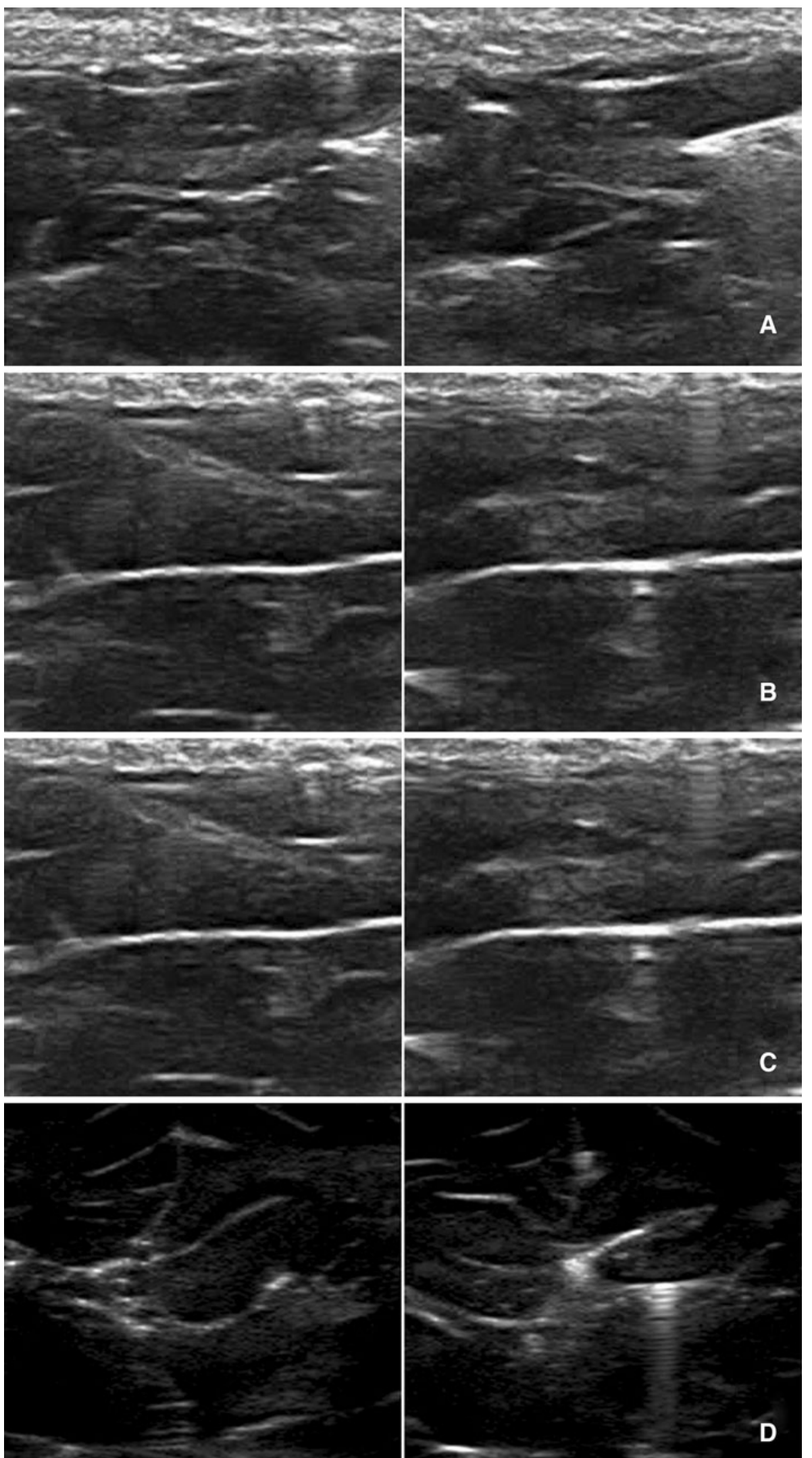
glioma samples cannot be treated as the equivalent of microglial cells that gradually replace neurons in Parkinson's disease. The exact structure, number and concentration of cells are different. Keeping in mind these limitations, one has to pay attention to striking sensibility of TCS in depicting brain tissue structure changes, especially connected with glial cells. TCS has been used for many years as an useful diagnostic tool in neuro-oncology. This method is especially useful in post-operative followup of patients with high-grade gliomas being, in experienced hands, one of the most sensitive techniques in identification of residual tumour (Becker et al. 1999). GII and GIV glioma samples, we have used for the experiment, were clearly visible. Applying this finding to Parkinson's disease there is a neuroinflammatory glial response present in parkinsonian brains from the very beginning of neurodegenerative process. The relative number and activity of glial cells appear to be stable during disease progression (Ouchi et al. 2005), which corresponds well to the stable area of SN hyperechogenicity observed in 5-years followup (Berg et al. 2005). We hypothesize that gliosis is, at the present level of knowledge, the simplest and most satisfactory biological explanation of SN hyperechogenicity in neurodegenerative diseases. The role of other considerable factors such as changes in protein conformation or alfasynuclein aggregation remains to be elucidated.

To support our hypothesis the physics of ultrasound examination could be taken into account. Ultrasound echo is created due to reflection and scattering phenomena. As reflection occurs only on boundaries, it is scattering that actually produces tissue echogenicity. Magnitude of scattering produced echogenicity is proportional to the number of objects that actually interacts with ultrasound wave. Therefore, the ratio between the object size and the ultrasound wave length is the most important factor. As proteins and iron nanoparticles are much smaller than ultrasound wave length, other factors like the change in cell properties or arrangement seem to be much more significant. Keeping in mind all limitations and inaccuracies of animal model used, it seems to be more probable that hyperechogenicity of $\mathrm{SN}$ is caused rather by structural changes within the brain tissue than by an increase of iron concentration. Although our comparative studies are informative, still more experiments are needed to elucidate hyperechogenicity of SN in PD phenomena, particularly role of iron.

Conflict of interest The authors declare that they have no conflict of interest.
Open Access This article is distributed under the terms of the Creative Commons Attribution Noncommercial License which permits any noncommercial use, distribution, and reproduction in any medium, provided the original author(s) and source are credited.

\section{References:}

Becker G, Seufert J, Bogdahn U, Reichmann H, Reiners K (1995) Degeneration of substantia nigra in chronic Parkinson's disease visualized by transcranial color-coded real-time sonography. Neurology 45:182-184

Becker G, Hoffman E, Woydt M, Hulsmann U, Maurer M, Lindner A et al (1999) Postoperative imaging of high-grade gliomas: comparison of transcranial sonography, magnetic resonance imaging and computed tomography. Neurosurgery 44:469-477

Berg D, Becker G, Zeiler B, Tucha O, Hofmann E, Preier M et al (1999a) Vulnerability of the nigrostriatal system as detected by transcranial ultrasound. Neurology 53:1026-1031

Berg D, Grote C, Rausch WD, Maurer M, Wesemann W, Riederer P, Becker G (1999b) Iron accumulation in the substantia nigra in rats visualized by ultrasound. Ultrasound Med Biol 25:901-904

Berg D, Roggendorf W, Schröder U, Klein R, Tatschner T, Benz P et al (2002) Echogenicity of the substantia nigra: association with increased iron content and marker for susceptibility to nigrostriatal injury. Arch Neurol 59:999-1005

Berg D, Merz B, Reiners K, Naumann M, Becker G (2005) Five-year follow-up study of hyperechogenicity of the substantia nigra in Parkinson's disease. Mov Disord 20:383-385

Berg D, Godau J, Riederer P, Gerlach M, Arzberger T (2010) Microglia activation is related to substantia nigra echogenicity. J Neural Transm 117(11):1287-1292

Hughes AJ, Daniel SE, Ben-Shlomo Y, Lees AJ (2002) The accuracy of diagnosis of parkinsonian syndromes in a specialist movement disorder service. Brain 125:861-870

Ouchi Y, Yoshikawa E, Sekine Y, Fatatsubashi M, Kanno T, Ogusu T et al (2005) Microglial activation and dopamine terminal loss in early Parkinson's disease. Ann Neurol 57:168-175

Walter U, Niehaus L, Probst T, Benecke R, Meyer BU, Dressler D (2003) Brain parenchyma sonography discriminates Parkinson's disease and atypical parkinsonian syndromes. Neurology 60:74-77

Walter U, Dressler D, Wolters A, Wittstock M, Greim B, Benecke R (2006) Sonographic discrimination of dementia with Lewy bodies and Parkinson's disease with dementia. J Neurol 253:448-454

Wypijewska A, Gałązka-Friedman J, Bauminger ER, Wszołek ZK, Schweitzer KJ, Dickson DW et al (2010) Iron and reactive oxygen species activity in parkinsonian substantia nigra. Parkinsonism Relat Disord 16:329-333

Zecca L, Berg D, Arzberger T, Ruprecht P, Rausch WD, Musicco M et al (2005) In vivo detection of iron and neuromelanin by transcranial sonography: a new approach for early detection of substantia nigra damage. Mov Disord 20:1278-1285 